\title{
Performance Analysis of Sparse MIMO-OFDM Channel Estimation Using Spatial and Temporal Correlations
}

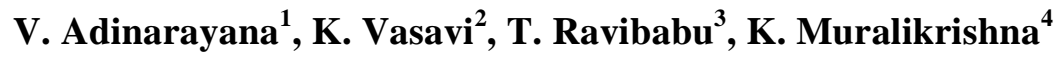 \\ ${ }^{1,2}$ Department of ECE, Vignan's Institute of information Technology, Visakhapatnam, Andhra Pradesh, India \\ ${ }^{3}$ Department of ECE, Sanketika Vidya parishad Engineering College, Visakhapatnam, Andhra Pradesh, India \\ ${ }^{4}$ Department of ECE, Anil Neerukonda Institute Technology and Science, Visakhapatnam, Andhra Pradesh, India
}

\begin{abstract}
To improve accuracy of the channel, this paper proposes a parametric sparse multiple input multiple output (MIMO)-OFDM channel estimation scheme based on the finite rate of innovation (FRI) theory, whereby super-resolution estimates of path delays with arbitrary values can be achieved. For outdoor communication scenarios, where wireless channels are sparse in nature, path delays of different transmit-receive antenna pairs share a common sparse pattern due to the spatial correlation of MIMO channels. Meanwhile, the channel sparse pattern is nearly unchanged during several adjacent OFDM symbols due to the temporal correlation of MIMO channels. The proposed scheme performs better than existing schemes. Furthermore, by joint processing of signals associated with different antennas, the pilot overhead can be reduced under the framework of the FRI theory. Meanwhile, both the spatial and temporal correlations of wireless MIMO channels are exploited to improve the accuracy of the channel estimation.
\end{abstract}

Keywords: MIMO-OFDM, Super-resolution, sparse channel estimation, finite rate of innovation (FRI), spatial and temporal correlations

\section{Introduction}

In broadband wireless communications, MIMO (Multiple Input Multiple Output) OFDM becomes more efficient to achieve high data rate and better performance. Accurate and efficient channel estimation plays a key role in MIMO-OFDM wireless communications. Channel capacity of MIMO-OFDM system is increased by channel estimation. The increase in the demand for bandwidth and different high performance services opened the door for using multiple antennas at transmitter and receiver. The wireless channel properties are dynamic in nature as it is frequency selective and time-dependent. Multiple Input Multiple Output (MIMO)-OFDM is widely recognized as a key technology for future wireless communications due to its high spectral efficiency and superior robustness to multipath fading channels [1]. In general, there are two groups of channel estimation schemes for MIMO-OFDM system. The first one is nonparametric channel estimation scheme, which adopts orthogonal frequency-domain pilots or orthogonal time-domain training sequences to convert the channel estimation in MIMO systems to that in single antenna systems [2]. However, such scheme suffers from high pilot overhead when the number of transmit antennas increases. The second category is parametric channel estimation scheme, which exploits the sparsity of wireless channels to reduce the pilot overhead [3], [4]. The parametric scheme is more favorable for future wireless systems as it can achieve higher spectral efficiency. However, path delays of sparse channels are assumed to be located at the integer times of the sampling period [3], which is usually unrealistic in practice. In this work, a more practical sparse MIMO-OFDM channel estimation scheme based on spatial and temporal correlations of sparse wireless MIMO channels is proposed to deal with arbitrary path delays [9]. Third, we reduce the pilot overhead by using the finite rate of innovation (FRI) theory [8], which can recover the analog sparse signal with very low sampling rate, as a result, the average pilot overhead per antenna only depends on the channel sparsity level instead of the channel length.

In this paper, an algorithm is presented for the estimation of sparse Multiple Input Multiple Output (MIMO)-OFDM channel estimation [11], [12]. The most significant taps of sparse channel are obtained by analysis of pilot bits of received signal. Along with this, spatial and temporal correlations of wireless channels are used to improve the channel estimation accuracy and super-resolution estimate of path delays with arbitrary values can be achieved using finite rate of innovation theory.

\section{Sparse Channel Model}

The following characteristics of MIMO are considered:

\subsection{Channel Sparsity}

LTE resolves the individual propagation paths from transmitters to receivers which results in a channel impulse response showing only a few peaks and many zeros which can be stated as a sparse signal [9]. In an outdoor environment CIR is normally sparse due to many significant scatterers. For an $N_{t} \times N_{r}$ MIMO system, the CIR $h^{i, j}(t)$ between the $i$ th transmit antenna and $j$ th receive antenna can be modeled as 
International Journal of Science and Research (IJSR)

ISSN (Online): 2319-7064

Index Copernicus Value (2013): 6.14 | Impact Factor (2014): 5.611

$h^{i, j}(t)=\sum_{P=1}^{P} \alpha_{P}^{(i, j)} \delta\left(t-\tau_{P}^{(i, j)}\right), \quad 1 \leq i \leq N_{t}, 1 \leq j \leq N_{r}$

Where

$\delta$ (.) is the Dirac Function, $\mathrm{P}$ is total number of resolvable propagation paths, and $\alpha_{P}{ }^{(i, j)}$ and $\tau_{P}{ }^{(i, j)}$ denote the path gain and path delay of the pth path, respectively.

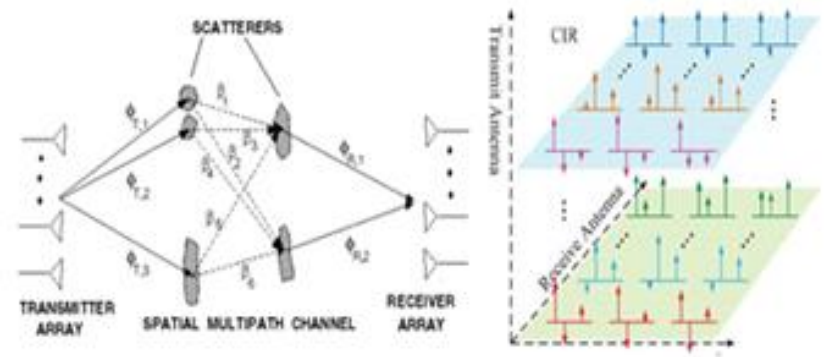

Figure 1: Spatial and temporal correlations of MIMO channels

\subsection{Spatial Correlation}

As compared to the long signal transmission distance, the scale of the transmit/receive antenna array is impeccably small, therefore channels of various transmit-receive antenna pairs share very similar scatterers. Meanwhile, for many communication systems, the path delay difference from the same scatterer is very much less than the system sampling period. Hence, CIRs of different transmit-receive antenna pairs have a common sparse pattern, even though the corresponding path gains may be quite different.

\subsection{Temporal Correlation}

Path delays of CIRs for several adjacent OFDM symbols are almost unchanged which can be equivalently stated as a common spars e pattern of CIRs because of the temporal correlation of MIMO channels. In wireless channels, the path gains vary continuously compared to the path delays. Hence, the channel sparse pattern is almost unchanged for several adjacent OFDM symbols as well as the path gains are also correlated [10].

\section{Super Resolution Sparse MIMO-OFDM Channel Estimation}

A pilot pattern is briefly introduced based on which a super resolution sparse MIMO-OFDM channel estimation is applied. Also, the required number of pilots is discussed under the framework of FRI theory.

\subsection{Pilot Pattern}

The pilot pattern used is shown in figure.3, $\mathrm{P}$ pilots are spaced equidistant to each other such that there is a pilot interval $\mathrm{G}$ between each other in the Frequency domain. In the meantime, each pilot is assigned with a pilot index 1 such that $0 \leq l \leq N_{P}-1$, whichis ascending with the increase of the subcarrier index [10]. Furthermore, MIMO channels are associated with different transmit antennas, each antenna uses a unique subcarrier index initial phase $\theta_{i}$ for $1 \leq i \leq N_{t}$ and $\left(N_{t}-1\right) N_{P}$ zero subcarrier to ensure the orthogonality of pilots. Therefore, for the $i^{\text {th }}$ transmit antenna, the subcarrier index of the $l^{\text {th }}$ pilot is

$$
I_{\text {pilot }}^{i}(l)=\theta_{i}+l G, 0 \leq l \leq N_{P}-1
$$

Consequently, the total pilot overhead per transmit antenna is

$$
N_{P_{-} \text {total }}=N_{t} N_{P}
$$

\subsection{Spatial and Temporal Correlation}

As the wireless channel is sparse by nature and the small scale of various transmit/receive antennas is negligible with respect to the large signal transmission distance, CIRs of multiple pairs of transmit-receive antennas have the common path delays, which is correspondingly interpreted as a common sparse pattern of CIRs owing to the spatial correlation of the MIMO channels [10].

i,e $\tau_{P}^{(i, j)}=\tau_{P}$ and $V^{(i, j)}[l]=V[l]$ for $1 \leq p \leq P, 1 \leq i \leq$ $N_{t}, 1 \leq j \leq N_{r}$, Hence, by utilizing such spatially common sparse pattern distributed among Mt transmit antennas and Mr receiver antennas pertaining to the $i^{\text {th }}$ transmit antenna, the channel frequency response is given by

Where

$$
\widehat{H}=V A+W
$$

$\widehat{H}$ is size of $N_{P} \times N_{t} N_{r}, \mathrm{~V}$ is a Vander monde matrix of size $N_{P} \times N_{P}$, A is path gain of size $N_{P} \times N_{r}$ and $N$ is additive white Gaussian noise. Comparing (1) with the traditional direction-of-arrival (DOA) problem it's been observed that these two are mathematically equivalent. Explicitly, the classical DOA problem is to commonly estimate the DOAs of the $\mathrm{M}$ sources from a group of time-domain quantities, which are acquired from the $N_{P}$ sensors outputs at $N_{t} \times N_{r}$ discrete points in time. On the contrary, the path delays of $\mathrm{N}$ multipath in (1) are estimated from a group of frequencydomain quantities, which are obtained from $N P$ pilots of $N_{t} \times N_{r}$ discrete pairs of antenna.

The Total Least Square-Estimating Signal Parameters via Rotational Invariance Techniques (TLS-ESPRIT) algorithm is used to estimate path delays with arbitrary values by applying it to (1). Additionally, the temporal correlation of wireless channels is also exploited to enhance the precision of the channel estimation. Initially, path delays of CIRs for various adjacent OFDM symbols are almost unaltered which is similarly, made reference to as a common sparse pattern of CIRs because of the temporal correlation of MIMO channels. Furthermore, path gains throughout adjacent OFDM symbols are also correlated pertaining to the temporal continuity of the CIR. Also various adjacent OFDM symbols are also correlated. Hence, noise is decreased which in turn improves the accuracy of channel estimation.

\section{Volume 5 Issue 1, January 2016}




\section{International Journal of Science and Research (IJSR)}

ISSN (Online): 2319-7064

Index Copernicus Value (2013): 6.14 | Impact Factor (2014): 5.611

\subsection{Pilot Overhead}

Based on the FRI theory it is stipulated that CIRs of $N_{t} \times N_{r}$ transmit-receive antenna pairs are similar to the $N_{t} \times N_{r}$ semi period sparse subspaces, and the $N_{P}$ pilots are similar to the $\tau_{p}$ multichannel filters. Hence, with respect to the FRI theory, the least required number of pilots for a transmit antenna is $\tau_{P}=2 Q$ (i.e., $Q$ is the sparsity level) in a noiseless environment. On the contrary, for the non-parametric channel estimation techniques the necessary number of pilots highly depend upon channel length $\mathrm{L}$, where as the super resolution sparse parametric scheme only requires $2 Q$ pilots where $Q$ $<<l$ due to AWGN channel [10].

\section{Simulation Results}

A simulation results are carried out by compare the performance of the proposed scheme with those of the existing methods for MIMO-OFDM systems. The conventional schemes were selected for nonparametric channel estimation schemes like comb-type pilot and timedomain training based orthogonal pilot (TTOP), and parametric scheme like time-frequency joint (TFJ) channel estimation. Consider the system parameters as follows: The carrier frequency is $f_{c}=1 \mathrm{GHz}$, the system bandwidth is $f_{s}=10 \mathrm{MHz}$, the size of the OFDM symbol is $\mathrm{N}=4096$, and $\mathrm{Ng}$ $=256$ is the guard interval length, and maximum delay spread is $25.6 \mu \mathrm{s}$. The International Telecommunication Union Vehicular B (ITU-VB) channel model with the maximum delay spread $20 \mu$ s and the number of paths $\mathrm{P}=6$ also considered.

In Fig. 2 compares the mean square error (MSE) performance of different channel estimation schemes. Both the static ITUVB channel and the time-varying ITU-VB channel with the mobile speed of $90 \mathrm{~km} / \mathrm{h}$ in a $4 \times 4$ MIMO system were considered. The comb-type pilot based scheme used $N_{p}=256$ pilots, the TTOP scheme used $N_{p}=64$ pilots with $T$ adjacent OFDM symbols for training, where $\mathrm{T}=4$ for the time-varying channel and $\mathrm{T}=8$ for the static channel to achieve better performance, the TFJ scheme adopted time-domain training sequences of 256-length and $N_{p}=64$ pilots, and our proposed scheme used $N_{p}=64$ pilots with $\mathrm{R}=4$ for fair comparison. From Figure we can observe that the conventional parametric TFJ scheme is inferior to the other three schemes obviously. Meanwhile, for static ITU-VB channel, the MSE performance of the proposed parametric scheme is more than $2 \mathrm{~dB}$ and 5 $\mathrm{dB}$ better than the TTOP and comb-type pilot based schemes, respectively.

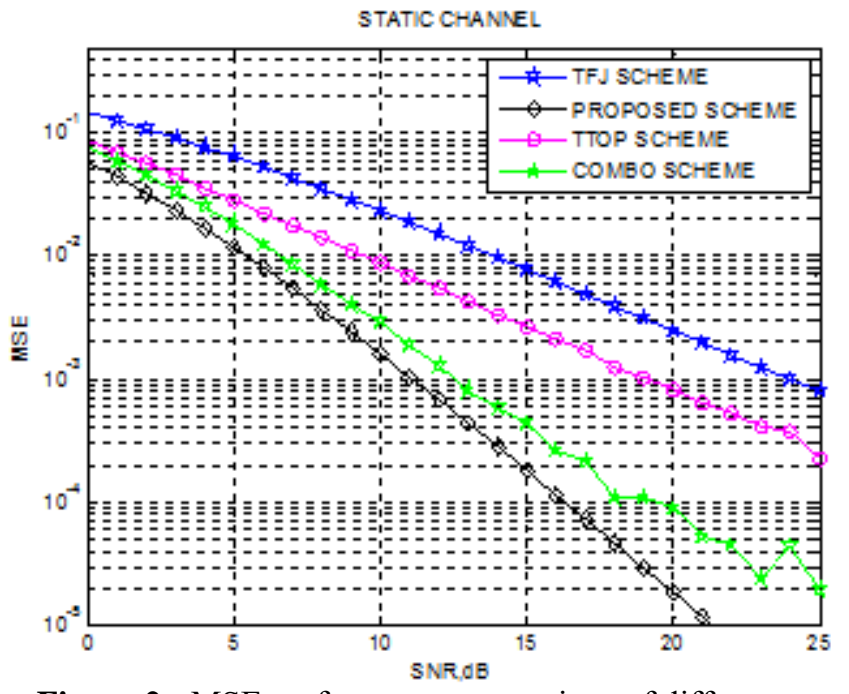

Figure 2 : MSE performance comparison of different schemes in a $4 \times 4$ MIMO system. (a) Static channel. (b) Timevarying Channel with the mobile speed of $90 \mathrm{~km} / \mathrm{h}$.

Moreover, for the time-varying ITU-VB channel, the superior performance of our proposed parametric scheme to conventional nonparametric schemes is more obvious. The existing sparse channel estimation scheme [4] does not work, because path delays may not be located at the integer times of the sampling period for practical channels. The TTOP scheme works well over static channels, but it performs poorly over fast time-varying channels, since it assumes that the channel is static during the adjacent OFDM symbols. Finally, the comb-type pilot based scheme performs worse than our proposed scheme, and it also suffers from much higher pilot overhead.

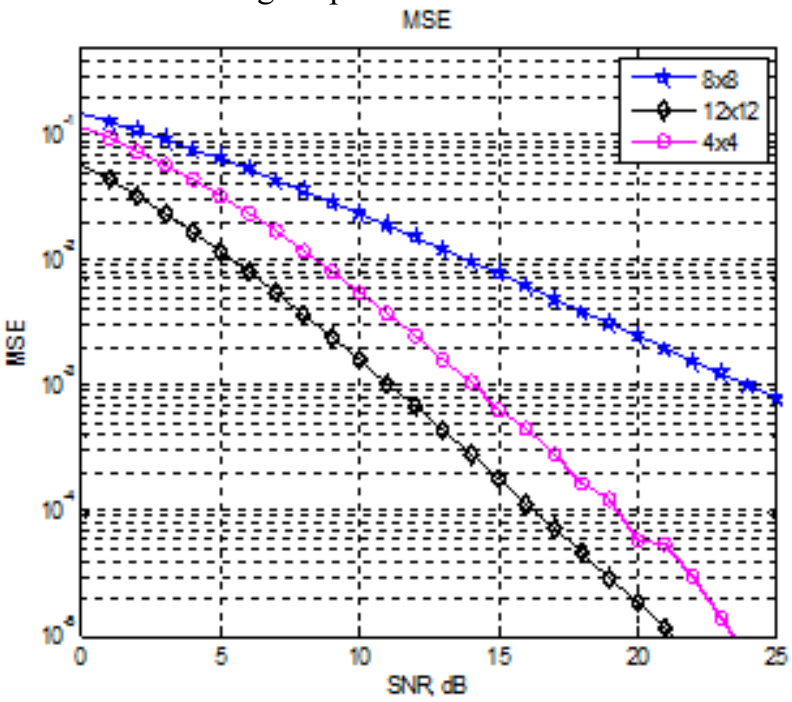

Figure 3 : MSE performance of the Proposed scheme in $4 \times 4$, $8 \times 8$, and $12 \times 12$ MIMO Systems

In Figure 3 compares the MSE performance of the proposed scheme in $4 \times 4,8 \times 8$, and $12 \times 12$ MIMO systems. We can observe that the MSE performance of the proposed scheme in $12 \times 12$ MIMO system is superior to that in $8 \times 8$ MIMO system by $5 \mathrm{~dB}$ with the same $N_{p}$ and outperforms that in $4 \times 4$ 


\section{International Journal of Science and Research (IJSR) \\ ISSN (Online): 2319-7064 \\ Index Copernicus Value (2013): 6.14 | Impact Factor (2014): 5.611}

MIMO system with the reduced $N_{p}$. These simulations indicate that with the increased number of antennas, the MSE performance improves with the same $N_{p}$. equivalently, to achieve the same channel estimation accuracy; the required number of pilots $N_{p}$ can be reduced. As a result, the total pilot overhead $N_{p_{-}}$total in our proposed scheme does not increase linearly with the number of transmit antennas $N_{t}$ because the required $N_{p}$ reduces when $N_{t}$ increases accordingly.

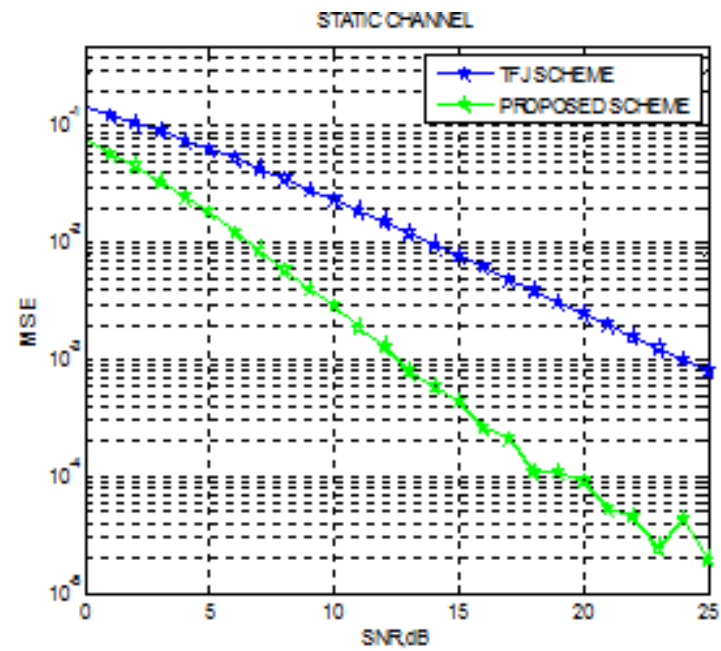

Figure 4 : MSE performance comparison of different schemes in a $4 \times 4$ MIMO system. (a) Static channel. (b) Timevarying Channel with the mobile speed of $90 \mathrm{~km} / \mathrm{h}$

The reason is that with the increased number of antennas, the dimension of the measurement matrix in the TLS-ESPRIT algorithm or the number of the sampling in the model of multiple filters bank [2] increases; thus, the accuracy of the path delay estimate improves accordingly. The superior performance of the proposed scheme is contributed by following reasons. First, the spatially common sparse pattern shared among CIRs of different transmit-receive antenna pairs is exploited in the proposed scheme, such that we can employ the TLS-ESPRIT algorithm to obtain super resolution estimations of path delays with arbitrary values. Meanwhile, the FRI theory indicates that the smallest required number of pilots is $N_{p}=2 P$ in a noiseless scenario. Therefore, the pilot overhead can be reduced as compared with conventional nonparametric schemes. Second, our scheme exploits the temporal correlation of wireless channels, namely, across several adjacent OFDM symbols, the sparse pattern of the CIR remains unchanged, and path gains are also correlated. Accordingly, by joint processing of signals of adjacent OFDM symbols based on (8), the effective noise can be reduced, and thus, the accuracy of the channel estimation is improved further.

\section{Conclusion}

Under the framework of the FRI theory, the required number of pilots in the proposed scheme is obviously less than that in nonparametric channel estimation schemes. Moreover, simulations demonstrate that the average pilot overhead per transmit antenna will be interestingly reduced with the increased number of antennas. The proposed super-resolution sparse MIMO channel estimation scheme exploits the sparsity as well as the spatial and temporal correlations of wireless MIMO channels. It can achieve super-resolution estimates of path delays with arbitrary values and has higher channel estimation accuracy than conventional schemes.

\section{References}

[1] QH Shi, Y Karasawa, Frequency-domain oversampling for OFDM systems: exploiting inter-carrier interference and multipath diversity. Int. Symp. Commun. Inform. Technol. 9, 1097-1101 (2009). doi:10.1109/ISCIT.2009.5341017

[2] L. Dai, J. Wang, Z. Wang, P. Tsiaflakis, and M. Moonen, Spectrum and energy-efficient OFDM based on simultaneous multi-channel reconstruction,"IEEE Trans. Signal Process., vol. 61, no. 23, pp. 6047-6059, Dec. 2013.

[3] P. L. Dragotti, M. Vetterli, and T. Blu, "Sampling moments and reconstructing signals of finite rate of innovation: Shannon meets Strang-Fix," IEEE Trans. Signal Process., vol. 55, no. 5, pp. 1741-1757, May 2007.

[4] L. Dai, Z. Wang, and Z. Yang, "Spectrally efficient timefrequency training OFDM for mobile large-scale MIMO systems," IEEE J. Sel. Areas Commun.,vol. 31, no. 2, pp. 251-263, Feb. 2013.

[5] Y. Barbotin and M. Vetterli, "Estimation of sparse MIMO channels with common support,"IEEE Trans. Commun., vol. 60, no. 12, pp. 3705-3716, Dec. 2012.

[6] I. Telatar and D. Tse, "Capacity and mutual information of wideband multipath fading channels,"IEEE Trans. Inf. Theory, vol. 46, no. 4, pp. 1384-1400, Jul. 2000.

[7] G. Stuberet al., "Broadband MIMO-OFDM wireless communications," Proc.IEEE, vol.92, no.2, pp. 271-294, Feb. 2004.

[8] I. Barhumi, G. Leus, and M. Moonen, "Optimal training design for MIMO-OFDM systems in mobile wireless channels,"IEEE Trans. Signal Process. vol. 3, no. 6, pp. 958-974, Dec. 2009.

[9] MR Raghavendra, $\mathrm{K}$ Giridhar, Improving channel estimation in OFDM systems for sparse multipath channels. IEEE Signal Process. Lett. 12(1), 52-55 (2005)

[10] G Taubock, F Hlawatsch, A compressed sensing technique for OFDM channel estimation in mobile environments: exploiting channel sparsity for reducing pilots. IEEE Acoustics Speech Signal Process. ICASSP-08, 2885-2888 (2008). doi:10.1109/ICASSP.2008.4518252.

[11]CR Berger, Z Wang, J Huang, S Zhou, Application of compressive sensing to sparse channel estimation. IEEE Commun. Mag. 48(11), 164-174 (2010)

[12] W. U. Bajwa, J. Haupt, A. M. Sayeed, and R. Nowak, "Compressed channel sensing: A new approach to estimating sparse multipath channels," Proc. IEEE, vol. 98, no. 6, pp. 1058-1076, Jun. 2010. 